\title{
State Distinction Based Identification Method for a Thermal Process
}

\author{
Ruifeng Zhang ${ }^{1}$, Xiaojun $\mathrm{Li}^{1}$,Xiankui Wen ${ }^{1}$, Hao Nan ${ }^{2}$, Yihao Zhang ${ }^{3, a}$ \\ ${ }^{1}$ Guizhou Electric Power Test and Research Institute,GuiYan,GuiZhou, 550000,China \\ ${ }^{2}$ Beijing Sifang Automation Co.Ltd,Beijing, 100084, China \\ ${ }^{3}$ North China Electric Power University.Beijing, 102206, China \\ aemail:euajin@126.com
}

Keywords: system identification; state distinction; least-square algorithm; thermal process

\begin{abstract}
At present, the closed-loop identification methods of nonlinear systems can not provide an satisfied performance. Generally, the step responses are performed to obtain the acceptable models of industrial processes. In consideration of this, the paper proposes a state distinction based identification method. According to the slopes of response, the approximated step responses can be distinguished from the living operating data and stored in the predefined array firstly. Based on the obtained data, the least square identification (LSI) is introduced to identify the linearized model of the process. The sum-square error (SSE) is used to determine the validity of the identified model. The example for a thermal process is employed to demonstrate the effectiveness of the proposed identification method. The results show that the identification method can provide an acceptable accuracy of the identified process in comparison with the results of step response.
\end{abstract}

\section{Introduction}

In China, thermal power plant is the dominant type used in the power system [1]. With the continuing increase of grid-connected wind power, the load demands of thermal power plants vary more frequently than ever before. In some local power system, the ration of wind power in the total capacity of the power system is more than 30 percent, which leads to the sawtooth wave of the curve of load demand [2]. In this situation, there are higher requirements of thermal power plant for the frequency stability of power system [3].

It is known that advanced control the theories (ACT's) can provide better performance than conventional proportion integration differentiation (PID) controller used, which benefits to improve the power system stability especially for frequency stability. However, those ACT's commonly depends on the high accurate models [4]. In consideration of this, a few of identification methods based on LSI have been deeply researched and widely reported [5]. The LSI has strict requirement for the input signals, which must cover the full operating range of the identified process. It is not easy to be obtained from living operating data. Alternatively, additional signals are added into control loop to simulate the plant. This maybe an effective way, while disturbances have been brought into the identified loop and the performance has been affected. The linearized model also can be identified from open-loop response. However, the operation must be interrupted by this method and the obtained model is a constant model, which is not adapted to the variation of operating conditions.

Considering above mentioned disadvantages of the LSI for nonlinear loops, this paper develops a state distinction based identification (SBDI) method, where the states are distinguished from the living operating data firstly. Based on the obtained input and output signals, the LSI is employed to identify the parameters of the equivalent linearized model. The paper is arranged as following: Section II, the state distinction method is introduced, where the slopes is used as the criteria of state distinction. The LSI method also employed to compute the parameters of identified model based on data from state distinction. An example for a 300MW thermal unit is used to show the effectiveness of the proposed SBDI method in Section III. Finally, the conclusions are drawn in Section IV. 


\section{The State Distinction Method}

The control systems commonly can be divided into two states, fault and normal states. For normal state, it also can be divided into two categories, such as the dynamic and steady states. There are a few reasons leaded to the faults of power system, such as software failures of control systems, disconnection of signal wire, and electrical failures. A detailed state distinction is displayed in Fig. 1. For that digital control systems have been widely used, the information of state distinction is generally obtained from the control system, such as distribute control system (DCS) and programmable logical controller (PLC).

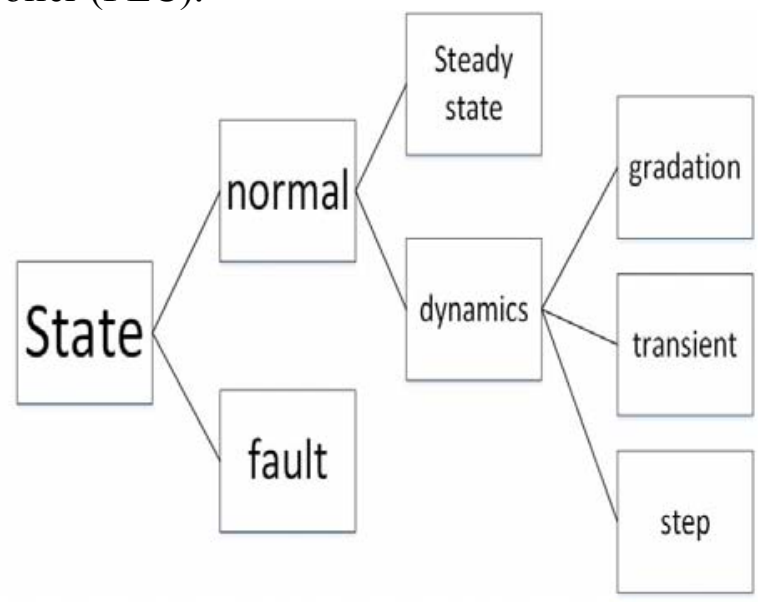

Figure 1. Control system state tree

\section{A. Real-time Data Segmentation}

Based on the system state distinction method, the extraction algorithm is extended to the online data segmentation algorithm, where the data can be obtained and computed point by point. In order to avoid the time accumulation effect of absolute error and threshold setting, the threshold value based on the error and SSE is used to determine the model validation. If the model is still valid, the accumulation sum of absolute error exceeds threshold value, a new model must be selected again compulsively.

The new data online segmentation algorithm provides the requirements of online data segmentation through these two steps.

First, in the present moment, the assumption that the linear model of the current state is represented by a vector, denoted as $L(i)\left[t_{0}(i), \hat{y}_{0}(i), t_{e}(i), \hat{y}_{e}(i)\right]$, where $t_{0}(i)$ is the initial moment of this section, $\hat{y}_{0}(i)$ is the initial value of this section, $t_{e}(i), \hat{y}_{e}(i)$ are the end of this section and the predictive value at the end of this section. The predictive value can be obtained according to the value read before and the current linear model:

$$
\hat{y}(t)=\frac{\hat{y}_{e}(i)-\hat{y}_{0}(i)}{t_{e}(i)-t_{0}(i)}\left(t-t_{0}(i)\right)+\hat{y}_{0}(i)
$$

(1) calculating error: $e(t)=y(t)-\hat{y}(t)$

(2) calculating SSE index of the current section: $\operatorname{SSE}(i)=\frac{1}{n} \sum_{j-t_{0}(i)}^{t} e(j)^{2}$

(3) calculating the number of SSE data: $n=\frac{t-t_{0}(i)}{T_{s}}$. We set two thresholds named $s_{1}, S_{2}$ for SSE.

There will be three cases comparing the values of SSE with $S_{1}$ and $S_{2}$ :

i. $S S E \leq S_{1}$, the current model is still valid and there is no need to update.

ii. $s_{1}<S S E \leq s_{2}, y_{t}$ is abnormal value, the moment and value should be stored in the outliner of the exception value group.

iii. $S S E \geq s_{2}$, at this time the current model $L(i)$ is no longer applicable. Put the moment and value 
into the exception value group, if the number of data sets in the array is greater than the minimum number of $n \_$out_max required for the model, the new linear model $L(i+1)$ will be calculated by the least square algorithm with the values in the group. Once the new linear function has been calculated, the SSE is set to zero, and the value of the array outliner is empty.

The algorithm based on the linear approximation used in the current time is no longer suitable for SSE, while the least square method is used to calculate new linear model which meets the requirement of SSE. This method judging whether the current linear relationship is still acceptable by SSE contains the cumulative difference between the observed values and the current model. It is very sensitive to data change to ensure that the theory can play a role in the face of data change.

\section{$B$. State Identification}

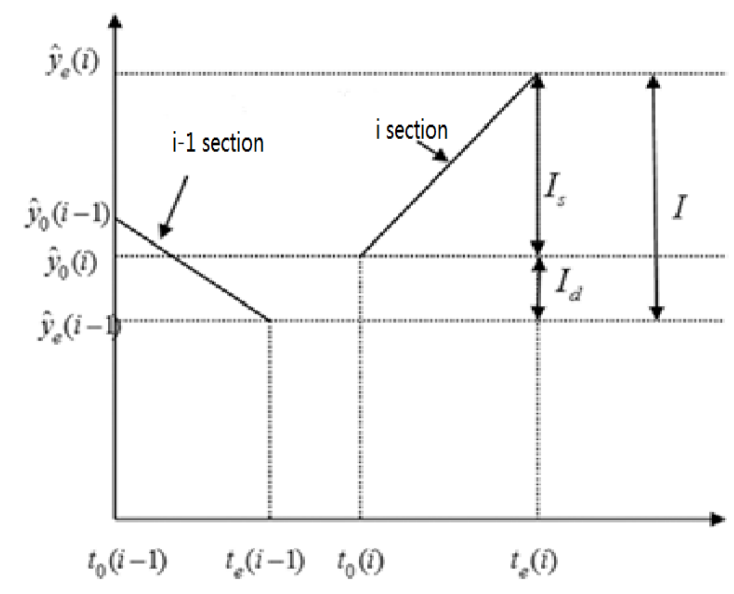

Figure 2. State identification illustration chart

State changes usually as the time when data model changes, as shown in Fig. 2, not only the information of this section, but also the information of last section is needed for state identification of i section, specially the relationship between the initial moment of this section and the end of last section is very important for state identification of this section. There are several numbers necessary for determining system state in two sections, as shown in Fig. 2, three numbers are calculated as follows:

Total increment, named $I$, is the difference between the predictive value of the end of $i$ section and the predictive value of the end of i-1 section:

$$
I=\hat{y}_{\varepsilon}(i)-\hat{y}_{\varepsilon}(i-1)
$$

Discontinuous increment, named $I_{d}$, is the difference between the predictive value of the initial moment of $i$ section and the predictive value of the end of $i-1$ section:

$$
I_{d}=\hat{y}_{0}(i)-\hat{y}_{e}(i-1)
$$

The segment increment, named $I_{s}$, is the difference between the predictive value of the end of $i$ section and the predictive value of the initial moment of $i$ section:

$$
I_{s}=\hat{y}_{e}(i)-\hat{y}_{0}(i)
$$

Therefore, we can define three slopes:

$$
\begin{aligned}
& k=\frac{I}{t_{e}(i)-t_{e}(i-1)} \\
& k_{s}=\frac{I_{s}}{t_{e}(i)-t_{0}(i)} \\
& k_{d}=\frac{I_{d}}{t_{0}(i)-t_{e}(i-1)}=\frac{I_{d}}{T_{s}}
\end{aligned}
$$

The three slopes include the running status information of the system, the reason why we choose the slopes, not increment, to identificate system state is that increment will accumulate accompanied with time increase so that there will be errors in state identification. What's more 
important is the consideration on choosing threshold. If increment is chosen, because each time interval is not consistent, it is difficult to determine the threshold. However, this problem can be solved by choosing slopes. The choice of the threshold equals the choice of a dip angle, if the angle is less than $\theta$, the system is in steady state, if the angle is greater than $\theta_{d}$, the system is transient, otherwise the system is gradient

Detailed judgement is shown in Fig. 3 of the discriminant tree. The state of i section can be obtained by comparing the three slopes with their thresholds, which provides a good basis for system identification. The threshold of the discontinuity slope is assumed as $\tan \left(\theta_{d}\right)$, by comparing with the threshold, the i section and i-1 section can be judged to be continuous or discontinuous. Noncontinuity means this section is one state of dynamic state, step, transient and continuity means current section is one state of steady, progress and taper in a certain. The total slope of the threshold is $\tan (\theta)$, the ratio of $\tan (\theta)$ and its threshold can be used to judge whether it is stable or not. When it is not in steady state, the $\operatorname{sign}$ of $\tan (\theta)$ can be used to judge whether it is increase or reduce. Threshold of this period's slope is $\tan \left(\theta_{s}\right)$, the ratio of the slope and its threshold can be used to judge whether it is step or not.

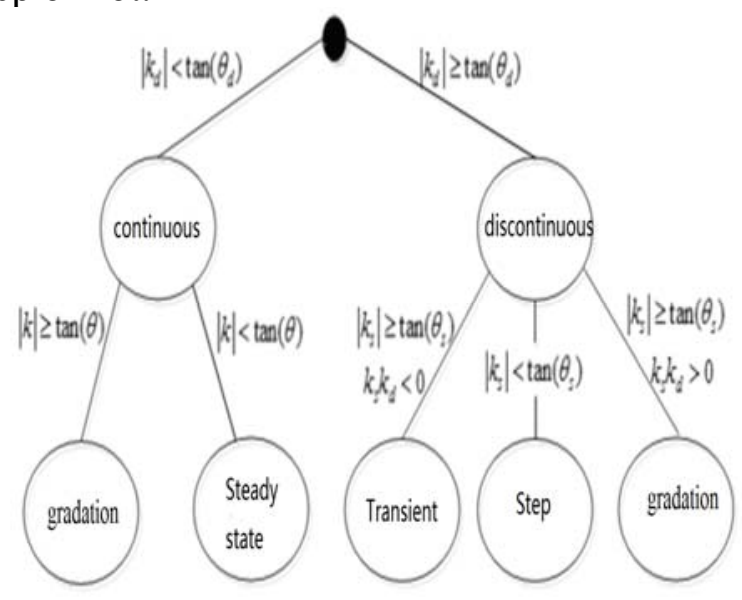

Figure 3. State discrimination tree

The flow chart of the online state discrimination is shown in Fig. 4, where three vectors named L,L1,L0, have four elements respective which are t0, y0 ,te and ye estimates, that is the vector representation of the linear model in last section. The values of $\mathrm{B}$ can be expressed differently to represent different states, such as $0,+1,+2,+3$, respectively, the state of steady, gradation, step, and transient. Where L1 is an accurate model for the $\mathrm{i}$ section, L0 is an accurate model for the $\mathrm{i}-1$ section. L, L1 equal indicating that the current model is still valid, the state remains unchanged, the $\mathrm{B}$ remains unchanged otherwise it means that the new section has been established and there is a need to re judge the current state and then to update B. It is worth noting that the model vector L read in Fig. 4 comes from the real-time results in Fig.2. The final output of the state representation matrix B is sent to the system identification module. Different system identification algorithms satisfy different states, only when the state is determined can the system identification be carried out. 


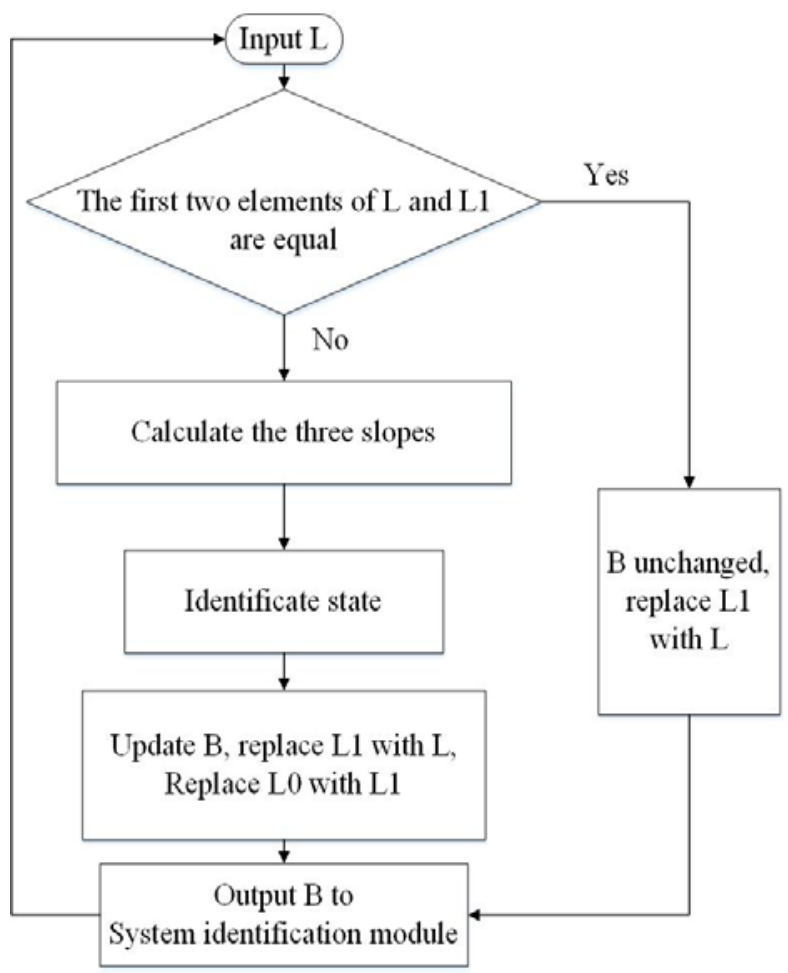

Figure 4. Program flow chart

\section{C. least square identification}

Least square method is proposed in Gauss's 'star movement orbit prediction research work' in 1795. The idea of least squares method is to calculate the difference between the actual value and the calculated value in many times. After square, the difference is multiplied by the accuracy to obtain a numerical value. Finally minimize the sum of those values.

For the SISO discrete stochastic systems, we describe it as:

$$
A\left(z^{-1}\right) z(k)=B\left(z^{-1}\right) u(k)+e(k)
$$

where

$\mathrm{u}(\mathrm{k})$ is the system input,

$\mathrm{z}(\mathrm{k})$ is the output,

$\mathrm{e}(\mathrm{k})$ is uncorrelated random noise whose mean value is 0 .

$$
\begin{aligned}
& A\left(z^{-1}\right)=1+a_{1} z^{-1}+a_{2} z^{-2}+\ldots+a_{n a} z^{-n a} \\
& B\left(z^{-1}\right)=1+b_{1} z^{-1}+b_{2} z^{-2}+\ldots+b_{n b} z^{-n b}
\end{aligned}
$$

From system (1), we can obtain least squares of system input and output

$$
z(k)=h^{T}(k) \theta+e(k)
$$

where $\mathrm{h}^{\mathrm{T}}(\mathrm{k})$ and $\mathrm{e}(\mathrm{k})$ are observable data vector and the identified parameter sets respectively:

$$
\begin{aligned}
& h^{T}(k)=[-z(k-1), \ldots,-z(k-n a), u(k), \ldots, u(k-n b)] \\
& q=\left[a_{1}, a_{2}, \ldots, a_{n a}, b_{1}, b_{2}, \ldots, b_{n b}\right]
\end{aligned}
$$

Notice that $\mathrm{z}(\mathrm{k})$ and $\mathrm{h}^{\mathrm{T}}(\mathrm{k})$ are data series, after measuring one data length, we can obtain system of linear equations formed by one equation, then minimize the following criterion function:

$$
\begin{aligned}
& J(\theta)=\sum_{k=1}^{i}\left[z(k)-h^{T}(k) \theta\right]^{2}=(Z-H \theta)^{T}(Z-H \theta) \\
& Z=[z(1), z(2), \cdots, z(l)]^{T}, H=\left[\begin{array}{l}
h^{T}(1) \\
h^{T}(2) \\
\vdots \\
h^{T}(l)
\end{array}\right]
\end{aligned}
$$

Take $\theta$ for estimated value of $J(\theta)=\min \theta$, the problem is equivalent to calculate the least square 
estimate whose parameter is $\theta$.

$$
\left.\frac{\partial J(\theta)}{\partial(\theta)}\right|_{\hat{\theta}}=\frac{\partial}{\partial(\theta)}(Z-H \theta)^{T}(Z-H \theta)=0
$$

From Eq. (6) and vector differential equation, we obtain $\left(H^{T} H\right)^{-1} H^{T} Z$, when $H^{T} H$ is regular matrix, we can obtain

$$
\hat{\theta}=\left(H^{T} H\right)^{-1} H^{T} Z
$$

Equation (7) is the Least Squares basic arithmetic primary calculation result.

$$
\left.\frac{\partial^{2} J(\theta)}{\partial \theta^{2}}\right|_{\hat{\theta}}=2 H^{T} H>0
$$

So $\hat{\theta}$ which satisfy Eq. (5) and $J(\theta)=\min \theta$ is unique.

Recursive calculation formula of the Least Squares basic arithmetic is as follows:

$$
\begin{aligned}
& \hat{\theta}(k)=\hat{\theta}(k-1)+K(k)\left[z(k)-h^{T}(k) \hat{\theta}(k-1)\right] \\
& K(k)=P(k-1) h(k)\left[h^{T}(k) p(k-1) h(k)+1\right]^{-1}(8) \\
& P(k)=\left[I-K(k) h^{T}(k)\right] P(k-1)
\end{aligned}
$$

where $\mathrm{k}$ is number of recursive, $\mathrm{I}$ is unit matrix.

\section{Open Loop Simulation Experiment}

Consider object $G_{0}=\frac{z+0.5}{Z^{2}-1.5 z+0.7}$, choose white noise as input signal for simulation identification. Fig. 5 shows the actual system output y (dashed line) and Y1 model (solid line) curve. Fig. 6 is the original model $G_{0}$ (dashed line) and identification model $G$ (solid line) Bode comparison chart.

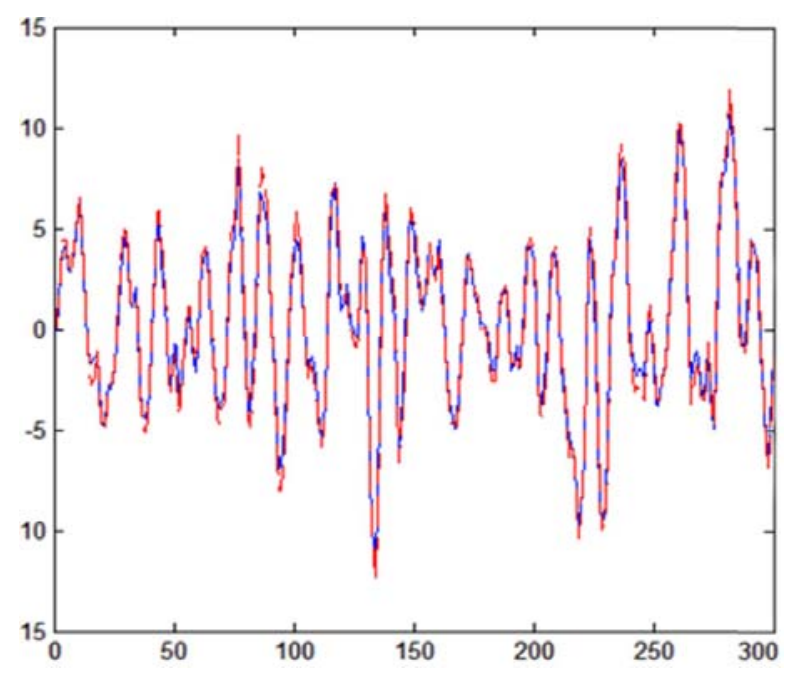

Figure 5. Open loop test identification and actual output comparison chart 


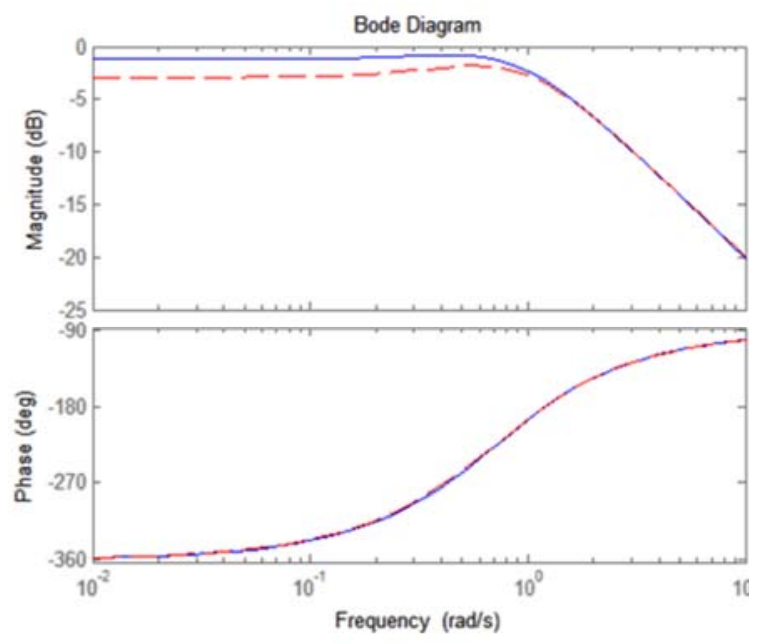

Figure 6. Open loop test identification and actual output Bode diagram

We analyze the status of the actual load data of the $330000 \mathrm{~kW}$ thermal power units from Dalate power plant 2. We take the data from 10:40:00 to 12:00:00 in 6th November, 2014, whose time interval is $1 \mathrm{~s}$ and amount is 4835 .

First step, according to the system state discrimination method based on the trend term extraction before, the data are fitted to get the curve trend as Fig. 7 shows. After algorithm processing, we can get the result in Fig.8. The 2500 data from 10:40:00 to 11:21:00 are used for identification, we can get the result in Fig. 9.

The dashed line is the actual output, the solid line is the output of the model for the identification in Fig.9, where $\mathrm{k}=1.7579, \mathrm{t}=265.1168, \tau=28$.

We can see that after choosing data suitable for identification through state discrimination in first step, we can improve the accuracy of identification in Fig. 6 and 7.

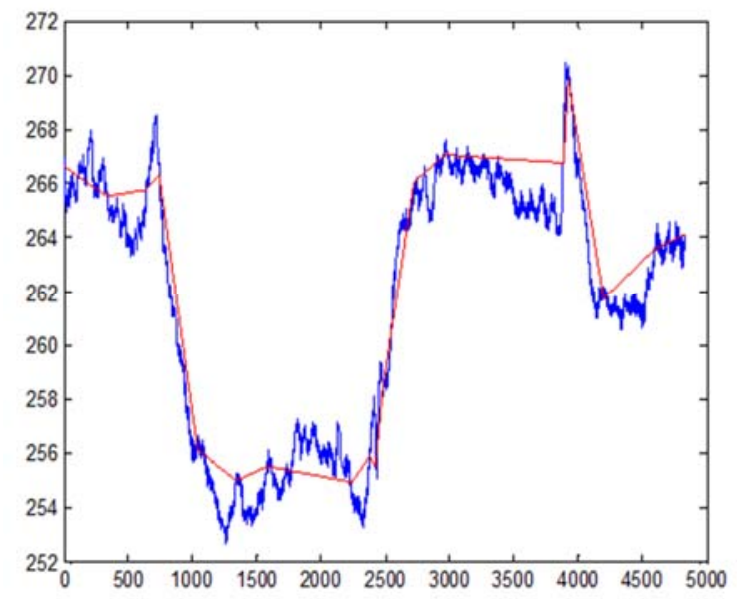

Figure 7. Load curve and fitting curve 


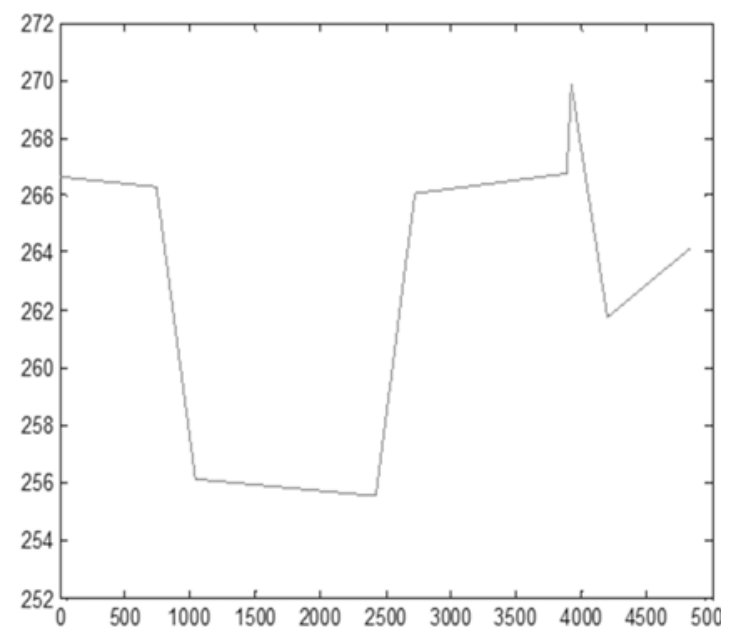

Figure 8. Algorithm processing diagram

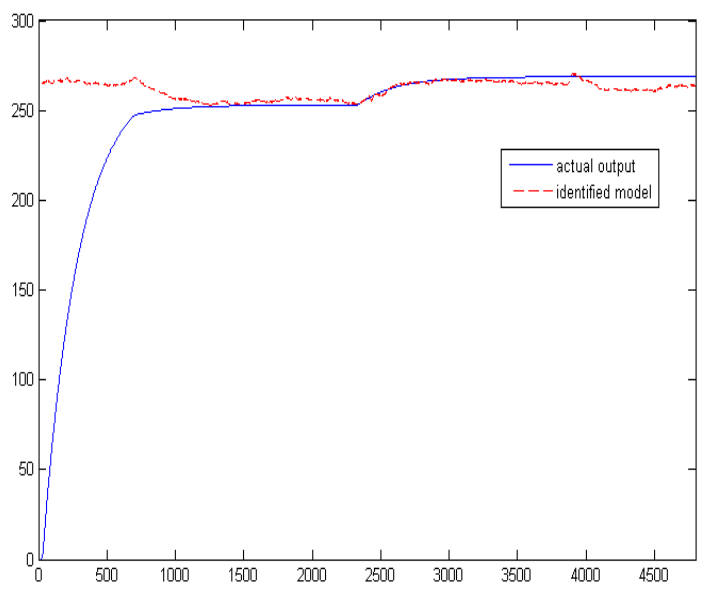

Figure 9. Comparison chart between actual output and identification output of 2500 data

\section{Conclusion}

In this paper, a system identification method based on state identification is proposed, which is aimed at the data of the real-time and historical data and identificate the state of the input data in the return channel. When the data trend is judged to be step state, the system identification of the data section can get more accurate system transfer function.

\section{References}

[1] Xiaoping Xu. Research on the algorithm of modular nonlinear system identification [D]. Xi'an University of Technology,2010.

[2] Blaabjerg F, Chen Z, Kjaer S B. Power electronics as efficient interface in dispersed power generation systems[J]. Power Electronics, IEEE Transactions on, 2004, 19(5): 1184-1194.

[3] QUAN Y, ZHANG W, XU X. Step response identification method for 2-order with time-delay system[J]. Control Theory \& Applications, 2002, 6: 028.

[4] PAN T, LI S. Adaptive PID control for nonlinear systems based on lazy learning[J]. Control Theory \& Applications, 2009, 10: 029.

[5] Guo H, Liu H P, Wang L. Method for selecting parameters of least squares support vector machines and application[J]. Journal of System Simulation, 2006, 18(7): 2033-2036. 
[6] Danghui Liu, Yuanwen Cai. System identification method and its application [M].Beijing: National Defence Industry Press.2010.

[7] HASSIBI B.,SAYED A.H., KAILATH T.:'Indefinite quadratic estimation and control:a unified approach to $\mathrm{H} 2$ and $\mathrm{H} 1$ theories'(SIAM, 1999).

[8] ZHANG H.S.,XIE L.H.:'Control and estimation for the systems with input/output delays' [J] (Springer-Verlag, 2007).

[9] KWAKERNAAK H.:'Optimal filtering in linear systems with time delays'[J],IEEE Trans.Autom Control,1967, 12,(2),pp.169-173.

[10]PETERSEN I.R., SAVKIN A.V.:'Robust Kalman filtering for signals and systems with large uncertainties' [J] (Birkhäuser,1999).

[11]Ding Feng,Chen Tongwen. Combined parameter and output estimation of dual-rate systems using an auxiliarymodel[J].Automatica,2004,(10):1739-1748.doi:10.1016/j.automatica.2004.05.001

[12]Ding Feng,Chen Tongwen. Parameter estimation of dual-rate stochastic systems by using an output error method[J].IEEE Transactions on Automatic Control,2005,(09):1436-1441.doi:10.1109/TAC.2005.854654 